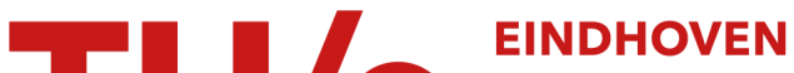 UNIVERSITY OF TECHNOLOGY
}

\section{On the synthesis of piecewise affine control laws}

Citation for published version (APA):

Bemporad, A., Heemels, W. P. M. H., \& Lazar, M. (2010). On the synthesis of piecewise affine control laws. In Proceedings of the 2010 IEEE International Symposium on Circuits and Systems (ISCAS), May 30 - June 2, 2010, Paris, France (pp. 3308-3311). Institute of Electrical and Electronics Engineers.

https://doi.org/10.1109/ISCAS.2010.5537905

DOI:

10.1109/ISCAS.2010.5537905

Document status and date:

Published: 01/01/2010

\section{Document Version:}

Publisher's PDF, also known as Version of Record (includes final page, issue and volume numbers)

\section{Please check the document version of this publication:}

- A submitted manuscript is the version of the article upon submission and before peer-review. There can be important differences between the submitted version and the official published version of record. People interested in the research are advised to contact the author for the final version of the publication, or visit the $\mathrm{DOI}$ to the publisher's website.

- The final author version and the galley proof are versions of the publication after peer review.

- The final published version features the final layout of the paper including the volume, issue and page numbers.

Link to publication

\section{General rights}

Copyright and moral rights for the publications made accessible in the public portal are retained by the authors and/or other copyright owners and it is a condition of accessing publications that users recognise and abide by the legal requirements associated with these rights.

- Users may download and print one copy of any publication from the public portal for the purpose of private study or research.

- You may not further distribute the material or use it for any profit-making activity or commercial gain

- You may freely distribute the URL identifying the publication in the public portal.

If the publication is distributed under the terms of Article $25 \mathrm{fa}$ of the Dutch Copyright Act, indicated by the "Taverne" license above, please follow below link for the End User Agreement:

www.tue.nl/taverne

Take down policy

If you believe that this document breaches copyright please contact us at:

openaccess@tue.nl

providing details and we will investigate your claim. 


\title{
On the Synthesis of Piecewise Affine Control Laws
}

\author{
A. Bemporad, Senior Member IEEE, W.P.M.H. Heemels, Member, IEEE, M. Lazar, Member IEEE
}

\begin{abstract}
Piecewise affine (PWA) control laws offer an attractive solution to real-time control of linear, nonlinear and hybrid systems. In this paper we provide a compact exposition of the existing state-of-the-art methods for the synthesis of PWA control laws using optimization-based methods.
\end{abstract}

\section{INTRODUCTION}

Piecewise affine (PWA) functions $f_{\mathrm{PWA}}: \mathbb{R}^{n} \rightarrow \mathbb{R}^{m}$ of the form [1], [2]

$$
f_{\mathrm{PWA}}(x):=F_{i} x+f_{i} \quad \text { if } \quad x \in \Omega_{i},
$$

where $\mathcal{I}=\{1, \ldots, s\}$ is a finite set of indices, $\left\{\Omega_{i}\right\}_{i \in \mathcal{I}}$ defines a partition of $\mathbb{R}^{n}$ with each $\Omega_{i}$ a polyhedron (not necessarily closed), $F_{i} \in \mathbb{R}^{m \times n}$ are matrices and $f_{i} \in \mathbb{R}^{m}$ are vectors for each $i \in \mathcal{I}$, have been largely used in modeling and control of dynamical systems. Applications of PWA functions include switched power converters [3], direct torque control of three-phase induction motors [4], automotive systems [5], paper flow in printers [6] and systems biology [7]. For discrete-time systems

$$
x(t+1)=f(x(t), u(t)),
$$

where $x(t) \in \mathbb{R}^{n}$ is the state vector and $u(t) \in \mathbb{R}^{m}$ is the control input vector, $t \in \mathbb{Z}_{+}$, the attractiveness of PWA control $u(t)=f_{\mathrm{PWA}}(x(t))$ is explained by various reasons. Firstly, PWA functions can be efficiently implemented on inexpensive hardware. Secondly, PWA functions are versatile and can approximate any nonlinear control law arbitrarily close. Thirdly, PWA control laws have a simple form that is close to affine/linear laws which (industrial) control engineers are familiar with. As a consequence of these interesting properties, there is a strong interest in synthesizing PWA controllers with desirable properties.

Several Lyapunov-based methods for synthesizing PWA laws exist in the literature [2], [6], [8]-[12]. However, one of the most appealing ways to construct PWA state feedback controllers for (constrained) linear and PWA system models is model predictive control (MPC). Indeed, recently it was shown that MPC, which requires on-line optimization, is in fact equivalent to an explicit PWA control law [13]-[16]. The goal of this paper is to provide a compact overview of

The authors are supported by the EU-FP7-ICT project MOBY-DIC, contract no. 248858 .

A. Bemporad is with Department of Information Engineering, University of Siena, Italy, E-mail: bemporad@dii.unisi.it.

W.P.M.H. Heemels is with the Mechanical Engineering Department, Eindhoven University of Technology, Eindhoven, The Netherlands, E-mail: m.heemels@tue.nl.

M. Lazar is with the Electrical Engineering Department, Eindhoven University of Technology, Eindhoven, The Netherlands, E-mail: m.lazar@tue.nl. the state-of-the-art MPC-based methods for the synthesis of PWA control laws.

\section{EXPLICIT MPC}

In this section we briefly recall the basic steps and ingredients to compute explicit solutions of MPC and their relations to PWA functions. The interested reader is referred to [16] for a more extensive survey on explicit MPC.

\section{A. Model predictive control}

In MPC the control action is obtained by solving a finite horizon open-loop optimal control problem at each sampling instant. Based on a discrete-time dynamic model one can obtain a prediction of the future states and outputs of the process given a control input sequence and initial conditions, which consist of the current (measured) state of the proces. Based on these predictions the best control sequence is chosen that satisfies input, state and output constraints and optimizes a performance index. This optimization yields a sequence of optimal control moves, but only the first move is applied to the process. At the next time step, the computation is repeated over a shifted time-horizon by taking the most recently available (measured) state information as the new initial condition of the optimal control problem. For this reason, MPC is also called receding horizon control.

The process to be controlled is usually modeled by the system of difference equations (2). We assume for simplicity that $f(0,0)=0$. The control and state sequences are requested to satisfy the constraints

$$
x(t) \in \mathcal{X}, u(t) \in \mathcal{U}
$$

$\forall t \in \mathbb{Z}_{+}$, where $\mathcal{U} \subseteq \mathbb{R}^{m}$ and $\mathcal{X} \subseteq \mathbb{R}^{n}$ are closed sets containing the origin in their interior ${ }^{1}$. Assuming that the control objective is to steer the state to the origin, MPC solves the following constrained regulation problem given a full measurement of the state $x(t)$ at the current time $t$ :

$$
\begin{aligned}
\mathcal{P}_{N}(x(t)): \min _{z} & \sum_{k=0}^{N-1} l\left(x_{k}, u_{k}\right)+F\left(x_{N}\right) \\
\text { s.t. } \quad & x_{k+1}=f\left(x_{k}, u_{k}\right), \quad k=0, \ldots, N-1 \\
& x_{0}=x(t), \\
& u_{k} \in \mathcal{U}, \quad k=0, \ldots, N-1 \\
& x_{k} \in \mathcal{X}, \quad k=1, \ldots, N-1 \\
& x_{N} \in \mathcal{X}_{N}, \\
& u_{k}=\kappa\left(x_{k}\right), \quad k=N_{u}, \ldots, N-1
\end{aligned}
$$

\footnotetext{
${ }^{1}$ Mixed constraints on $(x, u)$ can be treated as well, for instance to handle constraints on outputs with direct feedthrough $y(t)=f_{y}(x(t), u(t))$.
} 
where $z \in \mathbb{R}^{\ell}$, is the vector of optimization variables, $z=$ $\left[\begin{array}{llll}u_{0}^{\prime} & \ldots & u_{N_{u}-1}^{\prime}\end{array}\right]^{\prime}$ (' denotes the transpose), $\ell=m N_{u}, l$ is the stage cost, and the closed terminal set $\mathcal{X}_{N} \subseteq \mathcal{X}$, terminal $\operatorname{cost} F$, and terminal gain $\kappa$ are chosen to ensure closed-loop stability of the MPC scheme [17], [18]. At each time-step $t$, $x_{k}$ denotes the predicted state vector at time $t+k$, obtained by applying the input sequence $u_{0}, . ., u_{k-1}$ to model (2), starting from $x_{0}=x(t)$. The number $N \geq 1$ is the prediction horizon and $N_{u}$ is the control (input) horizon $\left(1 \leq N_{u} \leq N\right)$. In what follows we will also use " $\leq$ " for vector variables, to denote component-wise inequalities. Because $N$ is finite, if $f, l$ and $F$ are continuous and $\mathcal{U}$ is also compact the minimum in (4a) exists. At each time-step $t$ a solution to problem $\mathcal{P}_{N}(x(t))$ is found by solving the mathematical program

$$
\begin{array}{ll}
\min _{z} & h(z, x(t)) \\
\text { s.t. } & g(z, x(t)) \leq 0, g \in \mathbb{R}^{q}
\end{array}
$$

obtained from (4), yielding the optimal control sequence $z^{*}(x(t))$ (assuming for simplicity the uniqueness of the optimal sequence). Only the first input is applied to system (2), i.e.

$$
u(t)=z_{0}^{*}(x(t)),
$$

and the optimization problem (4) is repeated at time $t+1$, based on the new state $x(t+1)$ obtained from the process.

The basic MPC setup (4) can be specialized to different cases, depending on the prediction model, performance index, and terminal conditions used.

1) Linear model and quadratic cost: An optimal control problem (4) with quadratic costs and linear model is obtained when

$$
l\left(x_{k}, u_{k}\right)=x_{k}^{\prime} Q x_{k}+u_{k}^{\prime} R u_{k}, F\left(x_{N}\right)=x_{N}^{\prime} P x_{N}
$$

in (4a), where $Q=Q^{\prime} \geq 0, R=R^{\prime}>0$, and $P=P^{\prime} \geq 0$ are weight matrices and (4b) is given by

$$
f\left(x_{k}, u_{k}\right)=A x_{k}+B u_{k}
$$

$\kappa(x)=K x$ in $(4 \mathrm{~g}), \mathcal{U}, \mathcal{X}$ and $\mathcal{X}_{N}$ are polyhedral sets, e.g., $\mathcal{U}=\left\{u \in \mathbb{R}^{m}: u_{\min } \leq u \leq u_{\max }\right\}$. Then, by substituting $x_{k}=A^{k} x(t)+\sum_{j=0}^{k-1} A^{j} B u_{k-1-j}$, problem (5) becomes a quadratic program:

$$
\begin{aligned}
h(z, x(t)) & =\frac{1}{2} z^{\prime} H z+x^{\prime}(t) C^{\prime} z+\frac{1}{2} x^{\prime}(t) Y x(t) \\
g(z, x(t)) & =G z-W-S x(t),
\end{aligned}
$$

where $H=H^{\prime}>0$ and $C, Y, G, W, S$ are matrices of appropriate dimensions [13].

2) Linear model and linear cost: Let $\infty$ - or 1 -norms be used to measure performance

$$
l\left(x_{k}, u_{k}\right)=\left\|Q x_{k}\right\|_{p}+\left\|R u_{k}\right\|_{p}, F\left(x_{N}\right)=\left\|P x_{N}\right\|_{p},
$$

where $p=1, \infty, R \in \mathbb{R}^{n_{R} \times m}, Q \in \mathbb{R}^{n_{Q} \times n}, P \in \mathbb{R}^{n_{P} \times n}$, and use the same setup for (4b)-(4g) as in Section IIA.1. In case of $\infty$-norms, by introducing auxiliary variables $\epsilon_{0}^{u}, \ldots, \epsilon_{N-1}^{u}, \epsilon_{1}^{x}, \ldots, \epsilon_{N}^{x}$ satisfying $\epsilon_{k}^{u} \geq\left\|R u_{k}\right\|_{\infty}$ $(k=0, \ldots, N-1), \epsilon_{k}^{x} \geq\left\|Q x_{k}\right\|_{\infty}(k=1, \ldots, N-1)$, $\epsilon_{N}^{x} \geq\left\|P x_{N}\right\|_{\infty}$, or, equivalently,

$$
\begin{aligned}
& \epsilon_{k}^{u} \geq \pm R^{i} u_{k}, i=1, \ldots, n_{R}, \quad k=0, \ldots, N-1 \\
& \epsilon_{k}^{x} \geq \pm Q^{i} x_{k}, i=1, \ldots, n_{Q}, \quad k=1, \ldots, N-1 \\
& \epsilon_{N}^{x} \geq \pm P^{i} x_{N}, i=1, \ldots, n_{P}
\end{aligned}
$$

where the superscript $i$ in (11) denotes the $i$ th row, problem (4) can be mapped into the linear program [19]

$$
\begin{aligned}
& h(z, x(t))=\overbrace{1 \ldots 1}^{N} \overbrace{1 \ldots 1}^{N} \overbrace{0 \ldots 0}^{N} \overbrace{0}^{m N_{u}} z \\
& g(z, x(t))=G z-W-S x(t),
\end{aligned}
$$

where $z \triangleq\left[\begin{array}{lllllllll}\epsilon_{0}^{u} & \ldots & \epsilon_{N-1}^{u} & \epsilon_{1}^{x} & \ldots & \epsilon_{N}^{x} & u_{0}^{\prime} & \ldots & u_{N_{u}-1}^{\prime}\end{array}\right]^{\prime}$ is the optimization vector, and $G, W, S$ are obtained from weights $Q, R, P$, model matrices $A, B,(11)$, constraint sets $\mathcal{U}, \mathcal{X}, \mathcal{X}_{N}$, and gain $K$. The case of 1 -norms can be treated similarly by introducing slack variables $\epsilon_{i k}^{u} \geq \pm R^{i} u_{k}, \epsilon_{i k}^{x} \geq$ $\pm Q^{i} x_{k}, \epsilon_{i N}^{x} \geq \pm P^{i} x_{N}$. This reformulation extends beyond $1 / \infty$-norms to any convex piecewise affine cost $l, F$, that, thanks to the result of [20], can be rewritten as the max of a finite set of affine functions.

The use of linear programming in optimization-based control dates back to the early sixties [21].

3) Hybrid model and linear or quadratic costs: The MPC setup also extends to the case in which (4b) (and thus also (2)) is a hybrid dynamical model [22] of the form

$$
\begin{array}{r}
x_{k+1}=f\left(x_{k}, u_{k}, \delta_{k}, \zeta_{k}\right)=A x_{k}+B_{1} u_{k}+B_{2} \delta_{k}+B_{3} \zeta_{k} \\
E_{2} \delta_{k}+E_{3} \zeta_{k} \leq E_{1} u_{k}+E_{4} x_{k}+E_{5},
\end{array}
$$

involving both real and binary variables, denoted as the Mixed Logical Dynamical (MLD) model [23], where $x_{k} \in$ $\mathbb{R}^{n_{c}} \times\{0,1\}^{n_{b}}$ is the state vector, $u_{k} \in \mathbb{R}^{m_{c}} \times\{0,1\}^{m_{b}}$ is the input vector, and $\zeta_{k} \in \mathbb{R}^{r_{c}}, \delta_{k} \in\{0,1\}^{r_{b}}$ are auxiliary variables implicitly defined by (13b) for any given pair $\left(x_{k}, u_{k}\right)$. Matrices $A, B_{i},(i=1,2,3)$, and $E_{i}(i=1, \ldots, 5)$ denote real constant matrices, and the inequalities (13b) must be interpreted component-wise. In [22] it was shown that this MLD model has strong equivalence relations to PWA models, linear complementarity models [24] and other hybrid models.

The associated finite-horizon optimal control problem based on quadratic costs takes the form (9) with $z=$ $\left[\begin{array}{lllllllll}u_{0}^{\prime} & \ldots & u_{N-1}^{\prime} & \delta_{0}^{\prime} & \ldots & \delta_{N-1}^{\prime} & \zeta_{0}^{\prime} & \ldots & \zeta_{N-1}^{\prime}\end{array}\right]^{\prime}$ and subject to the further restriction that some of the components of $z$ must be binary. The hybrid MPC problem maps into a Mixed-Integer Quadratic Programming (MIQP) problem when the quadratic costs (7) are used in (4a) [25], or a Mixed-Integer Linear Programming (MILP) problem when $\infty$ - or 1-norms are used as in (10) [26]. 


\section{B. Multiparametric programming}

Consider the following mathematical program

$$
\begin{array}{cl}
\mathcal{M P}(x): \min _{z} & f(z, x) \\
\text { s.t. } & g(z, x) \leq 0 \\
& A z+B x+d=0,
\end{array}
$$

where $z \in \mathbb{R}^{\ell}$ collects the decision variables, $x \in \mathbb{R}^{n}$ is a vector of parameters, $f: \mathbb{R}^{\ell} \times \mathbb{R}^{n} \rightarrow \mathbb{R}$ is the objective function, $g: \mathbb{R}^{\ell} \times \mathbb{R}^{n} \rightarrow \mathbb{R}^{q}, A$ is a $q_{e} \times \ell$ real matrix, $B$ is a $q_{e} \times n$ real matrix, and $d \in \mathbb{R}^{q_{e}}$. Problem (14) is referred to as a multiparametric programming problem. We are interested in characterizing the (optimal) solution of problem (14) a function of $x$ for a given polytopic set $X$ of parameters. The solution of a multiparametric problem is a quadruple $\left(V^{*}, Z^{*}, z^{*}, X_{f}\right)$, where $(i)$ the set of feasible parameters $X_{f}$ is the set of all $x \in X$ for which problem (14) admits a solution, $X_{f}=\left\{x \in X: \exists z \in \mathbb{R}^{\ell}, g(z, x) \leq\right.$ $0, A z+B x+d=0\}$; (ii) The value function $V^{*}: X_{f} \rightarrow \mathbb{R}$ associates with every $x \in X_{f}$ the corresponding optimal value of (14); ( iii) The optimal set $Z^{*}: X_{f} \rightarrow 2^{\mathbb{R}^{\ell}}$ associates to each parameter $x \in X_{f}$ the corresponding set of optimizers $Z^{*}(x)=\left\{z \in \mathbb{R}^{\ell}: f(z, x)=V^{*}(x), g(z, x) \leq\right.$ $0, A z+B x+d=0\}$ of problem (14); (iv) An optimizer function $z^{*}: X_{f} \rightarrow \mathbb{R}^{\ell}$ associates to each parameter $x \in X_{f}$ an optimizer $z \in Z^{*}(x)\left(Z^{*}(x)\right.$ is just a singleton if $\mathcal{M P}(x)$ is strictly convex).

By treating $x(t)$ as the vector of parameters, the QP problem arising from the linear MPC formulation of Section IIA.1 can be treated as the multiparametric QP (mpQP)

$$
\begin{aligned}
\mathcal{Q P}(x): V^{*}(x)=\frac{1}{2} x^{\prime} Y x+\min _{z} & \frac{1}{2} z^{\prime} H z+x^{\prime} F^{\prime} z \\
\text { s.t. } & G z \leq W+S x .
\end{aligned}
$$

In [13], the authors investigated the analytical properties of the mpQP solution, that are summarized by the following theorem.

Theorem II.1 Consider a multiparametric quadratic program with $H>0,\left[\begin{array}{cc}H & F \\ F^{\prime} & Y\end{array}\right] \geq 0$. The set $X_{f}$ of parameters $x$ for which the problem is feasible is a polyhedral set, the value function $V^{*}: X_{f} \mapsto \mathbb{R}$ is continuous, convex, and piecewise quadratic, and the optimizer $z^{*}: X_{f} \mapsto \mathbb{R}^{\ell}$ is piecewise affine and continuous.

The immediate corollary is that the quadratic MPC approach based on linear costs described in Section II-A.2 admits a continuous piecewise-affine explicit solution of the form (1).

By treating $x(t)$ as the vector of parameters, the linear MPC formulation of Section II-A.2 can be treated as the multiparametric LP (mpLP)

$$
\begin{aligned}
\mathcal{L P}(x): \min _{z} & c^{\prime} z \\
\text { s.t. } & G z \leq W+S x,
\end{aligned}
$$

where $z \in \mathbb{R}^{\ell}$ is the optimization vector, $x \in X \subset \mathbb{R}^{n}$ is the vector of parameters, $c, G, W, S$ are suitable constant matrices and $X$ is the set of parameters of interest.
Theorem II.2 [27] Consider the mpLP problem (16). Then, the set $X_{f}$ is a convex polyhedral set, there exists an optimizer function $z^{*}: \mathbb{R}^{n} \rightarrow \mathbb{R}^{l}$, which is a continuous and piecewise affine function of $x$, and the value function $V^{*}: \mathbb{R}^{n} \rightarrow \mathbb{R}$ is a continuous, convex, and piecewise affine function of $x$.

The first methods for solving parametric linear programs appeared in 1952 in the master thesis published in [28], and independently in [29]. Since then, extensive research has been devoted to (multi)parametric analysis and programming, see the references in [20], [27], [27], [30]-[32]. Recently, there is a renewed interest in this field, mainly pushed by the application of mpLP in explicit MPC, see e.g. the recent survey [33].

As detailed in [26], the MPC formulation based on $\infty$-,1norms or quadratic costs subject to the MLD dynamics (13) can be solved explicitly by treating the optimization problem associated with MPC as a multiparametric mixed integer linear programming (mpMILP) problem or a multiparametric mixed integer quadratic programming (mpMIQP) problem, respectively. For further details regarding explicit PWA solutions to these types of mixed integer problems the interested reader is referred to [16].

\section{IMPLEMENTATION OF PWA CONTROL LAWS}

A PWA control (1) is essentially a lookup table of linear feedback gains. The right gain is selected on-line by finding the region $\Omega_{i}$ of the polyhedral partition where the current state $x(t)$ lies. This latter problem has been referred to as point-location problem. Note that if function $f_{P W A}$ is continuous, then one can always define the regions of the partitions as closed polyhedra $\Omega_{i}=\left\{x \in \mathbb{R}^{n}: H_{i} x \leq k_{i}\right\}$, as no ambiguity in the definition of $f_{P W A}$ would arise on possibly overlapping boundaries of different sets $\Omega_{i}, \Omega_{j}$. The most straightforward solution is to store all the $M$ polyhedra of the partition and carry out an on-line search through them until the right one is found. While this procedure is extremely easy to implement in a computer code, more efficient ways have been proposed for evaluating explicit MPC controllers. For instance, by exploiting the properties of multiparametric solutions, several solutions are proposed in the last few years, see e.g. [34]-[39] and the references therein.

From the hardware synthesis viewpoint, [40] showed that explicit MPC solutions can be implemented in an application specific integrated circuit (ASIC) with about 20,000 gates, leading to computation times in order of $1 \mu \mathrm{s}$. In the end, whether the explicit PWA form is preferable to the one based on on-line optimization depends on available CPU time, data memory, and program memory (see e.g. [41, Table II] for a comparison in the linear quadratic case).

\section{CONCLUSIONS}

In this short paper we gave a compact overview of recent MPC-based techniques for the synthesis of PWA control laws, together with pointers to the relevant literature for more details on this appealing topic. The potential of PWA controllers is enormous and we envision a tremendous growth 
of PWA control in the years to come in various application fields. Especially, if the implementation of PWA control laws on real-time hardware can be performed more efficiently and systematically, we foresee that PWA and MPC control will also be applied to faster systems that require sample frequencies in the order of $1 \mathrm{kHz}$ and above.

\section{REFERENCES}

[1] E. D. Sontag, "Nonlinear regulation: the piecewise linear approach," IEEE Transactions on Automatic Control, vol. 26, no. 2, pp. 346-357, 1981.

[2] M. Johansson, Piecewise linear control systems, ser. Lecture Notes in Control and Information Sciences. Springer, 2003, vol. 284.

[3] D. M. W. Leenaerts, "Further extensions to Chua's explicit piecewise linear function descriptions," International Journal of Circuit Theory and Applications, vol. 24, pp. 621-633, 1996.

[4] T. Geyer, G. Papafotiou, and M. Morari, "Model Predictive Control in Power Electronics: A Hybrid Systems Approach," in IEEE Conference on Decision and Control, Seville, Spain, 2005.

[5] A. Bemporad, P. Borodani, and M. Mannelli, "Hybrid control of an automotive robotized gearbox for reduction of consumptions and emissions," in Hybrid Systems: Computation and Control, ser. Lecture Notes in Computer Science, vol. 2623. Springer Verlag, 2003, pp. 81-96.

[6] J. de Best, B. Bukkems, M. van de Molengraft, W. Heemels, and M. Steinbuch, "Robust control of piecewise linear systems: a case study in sheet flow control," Control Engineering Practice, vol. 16, no. 8, pp. 991-1003, 2008 .

[7] S. Drulhe, G. Ferrari-Trecate, H. de Jong, and A. Viari, "Reconstruction of switching thresholds in piecewise-affine models of genetic regulatory networks," in Hybrid Systems: Computation and Control, ser. Lecture Notes in Computer Science, vol. 3927. Springer Verlag, 2006, pp. 184-199.

[8] D. Mignone, G. Ferrari-Trecate, and M. Morari, "Stability and stabilization of piecewise affine and hybrid systems: An LMI approach," in 39th IEEE Conference on Decision and Control, 2000, pp. 504-509.

[9] G. Ferrari-Trecate, F. A. Cuzzola, D. Mignone, and M. Morari, "Analysis of discrete-time piecewise affine and hybrid systems," Automatica, vol. 38, no. 12, pp. 2139-2146, 2002.

[10] J. Daafouz, P. Riedinger, and C. Iung, "Stability analysis and control synthesis for switched systems: A switched Lyapunov function approach," IEEE Transactions on Automatic Control, vol. 47, pp. 18831887, 2002.

[11] M. Lazar and W. P. M. H. Heemels, "Global input-to-state stability and stabilization of discrete-time piece-wise affine systems," Nonlinear Analysis: Hybrid Systems, vol. 2, pp. 721-734, 2008.

[12] M. Lazar, W. P. M. H. Heemels, and A. R. Teel, "Lyapunov functions, stability and input-to-state stability subtleties for discrete-time discontinuous systems," IEEE Transactions on Automatic Control, vol. 54, no. 10, pp. 2421-2426, 2009.

[13] A. Bemporad, M. Morari, V. Dua, and E. Pistikopoulos, "The explicit linear quadratic regulator for constrained systems," Automatica, vol. 38, no. 1, pp. 3-20, 2002.

[14] A. Bemporad, F. Borrelli, and M. Morari, "Model predictive control based on linear programming - The explicit solution," IEEE Transactions on Automatic Control, vol. 47, no. 12, pp. 1974-1985, 2002.

[15] A. Bemporad, W. Heemels, and B. De Schutter, "On hybrid systems and closed-loop mpc systems," IEEE Trans. on Aut. Control, vol. 47, no. 5, pp. 863-869, 2002.

[16] A. Alessio and A. Bemporad, "A survey on explicit model predictive control," in Nonlinear Model Predictive Control: Towards New Challenging Applications, ser. Lecture Notes in Control and Information Sciences, D. R. L. Magni, F. Allgower, Ed., vol. $384 . \quad$ Berlin Heidelberg: Springer-Verlag, 2009, pp. 345-369.

[17] D. Mayne, J. Rawlings, C. Rao, and P. Scokaert, "Constrained model predictive control: Stability and optimality," Automatica, vol. 36, no. 6, pp. 789-814, June 2000.

[18] M. Lazar, W. P. M. H. Heemels, S. Weiland, and A. Bemporad, "Stabilizing model predictive control of hybrid systems," IEEE Transactions on Automatic Control, vol. 51, no. 11, pp. 1813-1818, 2006.

[19] A. Bemporad, F. Borrelli, and M. Morari, "Model predictive control based on linear programming - The explicit solution," IEEE Trans. Automatic Control, vol. 47, no. 12, pp. 1974-1985, 2002.
[20] M. Schechter, "Polyhedral functions and multiparametric linear programming," Journal of Optimization Theory and Applications, vol. 53, no. 2, pp. 269-280, May 1987.

[21] A. Propoi, "Use of linear programming methods for synthesizing sampled-data automatic systems," Automation and Remote Control, vol. 24, no. 7, pp. 837-844, 1963.

[22] W. P. M. H. Heemels, B. De Schutter, and A. Bemporad, "Equivalence of hybrid dynamical models," Automatica, vol. 37, pp. 1085-1091, 2001.

[23] A. Bemporad and M. Morari, "Control of systems integrating logic, dynamics, and constraints," Automatica, vol. 35, pp. 407-427, 1999.

[24] W. P. M. H. Heemels, J. M. Schumacher, and S. Weiland, "Linear complementarity systems," SIAM journal on applied mathematics, vol. 60, no. 4, pp. 1234-1269, 2000.

[25] A. Bemporad and M. Morari, "Control of systems integrating logic, dynamics, and constraints," Automatica, vol. 35, no. 3, pp. 407-427, 1999.

[26] A. Bemporad, F. Borrelli, and M. Morari, "Piecewise linear optimal controllers for hybrid systems," in American Control Conference, Chicago, IL, June 2000, pp. 1190-1194.

[27] T. Gal, Postoptimal Analyses, Parametric Programming, and Related Topics, 2nd ed. Berlin: de Gruyter, 1995.

[28] W. Orchard-Hays, "Notes on linear programming (part 6): the Rand code for the simplex method (sx4)," Rand Corporation, Tech. Rep. 1440, 1955.

[29] S. Gass and T. Saaty, "The computational algorithm for the parametric objective function," Naval Research Logistics Quarterly, vol. 2, pp. $39-45,1955$

[30] T. Gal and H. G. (Eds.), Advances in Sensitivity Analysis and Parametric Programming, ser. International Series in Operations Research \& Management Science. Kluwer Academic Publishers, 1997, vol. 6.

[31] T. Gal and J. Nedoma, "Multiparametric linear programming," Management Science, vol. 18, pp. 406-442, 1972.

[32] C. Filippi, "On the geometry of optimal partition sets in multiparametric linear programming," Department of Pure and Applied Mathematics, University of Padova, Italy, Tech. Rep. 12, June 1997.

[33] C. Jones, M. Baric, and M. Morari, "Multiparametric linear programming with applications to control," European Journal of Control, vol. 13, pp. 152-170, 2007.

[34] M. Baotić, F. B. A. Bemporad, and M. Morari, "Efficient on-line computation of constrained optimal control," SIAM Journal on Control and Optimization, vol. 47, no. 5, pp. 2470-2489, 2008.

[35] P. Tøndel, T. A. Johansen, and A. Bemporad, "Evaluation of piecewise affine control via binary search tree," Automatica, vol. 39, no. 5, pp. 945-950, May 2003.

[36] C. Jones, P. Grieder, and S. Raković, "A logarithmic-time solution to the point location problem for parametric linear programming," Automatica, vol. 42, no. 12, pp. 2215-2218, 2006.

[37] J. Spjøtvold, S. Rakovic, P. Tøndel, and T. Johansen, "Utilizing reachability analysis in point location problems," in Proc. 45th IEEE Conf. on Decision and Control, San Diego, CA, 2006, pp. 4568-4569.

[38] F. Christophersen, M. Kvasnica, C. Jones, and M. Morari, "Efficient evaluation of piecewise control laws defined over a large number of polyhedra," in Proc. European Control Conf., Kos, Greece, 2007, pp. 2360-2367.

[39] Y. Wang, C. Jones, and J. Maciejowski, "Efficient point location via subdivision walking with application to explicit MPC," in Proc. European Control Conf., Kos, Greece, 2007, pp. 447-453.

[40] T. A. Johansen, W. Jackson, R. Schrieber, and P. Tøndel, "Hardware synthesis of explicit model predictive controllers," IEEE Trans. Contr. Systems Technology, vol. 15, no. 1, pp. 191-197, 2007.

[41] A. Bemporad, "Model-based predictive control design: New trends and tools," in Proc. 45th IEEE Conf. on Decision and Control, San Diego, CA, 2006, pp. 6678-6683. 